\title{
Where is the extremal Kerr ISCO?
}

\author{
Ted Jacobson \\ Center for Fundamental Physics \\ Department of Physics, University of Maryland \\ College Park, MD 20742-4111, USA
}

\begin{abstract}
Although the circular photon orbit and ISCO for the Kerr black hole remain distinct from each other and from the horizon in the extremal spin limit on a constant Boyer-Lindquist time slice, on a horizon-crossing slice they both coincide with the null generators of the horizon.
\end{abstract}

\section{Introduction}

In a classic paper on the physics of Kerr black holes, Bardeen, Press and Teukolsky [1] mentioned in passing a peculiar feature of the prograde circular photon and innermost stable (ISCO) orbits in the extremal limit: although the Boyer-Lindquist (BL) radial coordinates of these orbits both coincide with that of the horizon, their locations on a BL time slice are both distinct from the horizon and from each other. The photon orbit lies at a finite distance from the horizon, while the ISCO lies at an infinite distance from the horizon, and also at an infinite distance from any point with a greater value of the radial coordinate 1 This strange behavior, which will be explained in detail below, is tied to the fact that a BL time slice ends on the bifurcation surface (the intersection of the future and past horizons), and in the extremal limit the distance to the bifurcation surface becomes infinite. The geometry of the situation is illustrated in Fig. 1, which shows the causal structure of the equatorial plane of the Kerr spacetime in the region exterior to the future and past horizons.

On the other hand, the Kerr metric can of course also be described using time slices that cross the future horizon, like the Doran time slice in Fig. 1. For a rotating black hole that forms from gravitational collapse, only slices that cross the future horizon can remain in the stationary portion of the

\footnotetext{
${ }^{1}$ In Ref. [1] the marginally bound orbit was also discussed. In the extremal limit it lies a finite distance outside the photon orbit.
} 


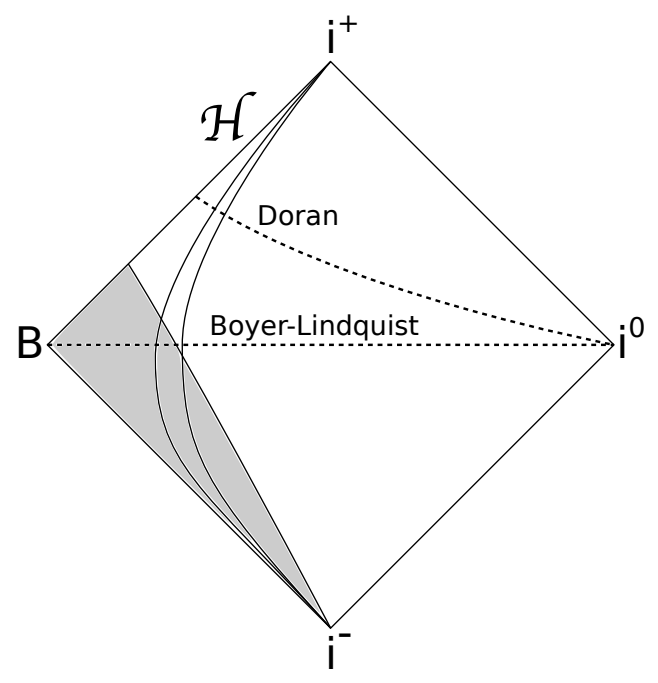

Figure 1: Carter-Penrose causal diagram of the equatorial plane of the Kerr spacetime exterior to the future and past horizons. Each point corresponds to a circle. $i^{ \pm}$are future and past timelike infinity, $i^{0}$ is spatial infinity, B is the bifurcation surface, and $\mathcal{H}$ is the future horizon. The dashed lines indicate Boyer-Linquist and Doran time slices as indicated. The two curves from $i^{-}$to $i^{+}$indicate the photon orbit (closer to $\mathcal{H}$ ) and the ISCO. The outer boundary of the gray region indicates the surface of matter that collapsed to form the black hole, so the gray region is not present in an astrophysical spacetime. In the extremal limit, the orbits both converge to the left edge of the diagram, and the proper distance to $B$ along the Boyer-Lindquist time slice diverges, whereas the distance to $\mathcal{H}$ along the Doran time slice remains finite.

spacetime. On such a slice, points with the same value of the radial and angular coordinates are certainly at the same location, so the extremal limit of the photon orbit and ISCO must in fact lie on the future horizon.

In occasional articles and private conversations I have encountered confusion generated by the result of Ref. [1]. Some of the confusion may arise from the way things are described in Ref. [1]. It is written there in section II that in the extremal limit, "it appears that the photon, marginally bound, and marginally stable orbits are coincident with the horizon. Appearances are deceptive!" It then goes on to say that in this limit the orbits "remain separated in proper radial distance" yet, after mentioning that some of these distances are infinite, adds parenthetically "The infinities are not physically important; an infalling particle follows a timelike curve, while the infinite distances are in a spacelike direction." This seems to clear things up but then in section III, after observing that the velocity of the ISCO measured 
relative to the locally non-rotating frame goes to $1 / 2$ and not 1 in the extremal limit, it is stated: "The point once again is that for $a=M$, the marginally stable orbit and the photon orbit are distinct." The aim of this note is to elucidate the compatibility of the two viewpoints, and to stress that on a horizon-crossing time slice the orbits in the limit are not distinct, and indeed coincide with the horizon generators.

\section{Boyer-Lindquist and Doran coordinates}

The Kerr black hole spacetime has both time translation symmetry and axial rotation symmetry. We will describe it here using both the BoyerLindquist and the Doran 2] coordinate systems. These have in common two coordinates, called $r$ and $\theta$, which are constant in the symmetry directions. The remaining two coordinates will be called $(t, \phi)$ for $\mathrm{BL}$ and $(\bar{t}, \bar{\phi})$ for Doran. The line element in these two coordinate systems takes the form

$$
\begin{gathered}
d s^{2}=-\left(1-\alpha^{2}\right) d t^{2}+\alpha^{-2} \beta^{2}\left(1-\beta^{2}\right)^{-1} d r^{2}-2 \alpha^{2} a \sin ^{2} \theta d t d \phi \\
+\rho^{2} d \theta^{2}+\left(r^{2}+a^{2}+\alpha^{2} a^{2} \sin ^{2} \theta\right) \sin ^{2} \theta d \phi^{2} \\
=-d \bar{t}^{2}+( \\
\left.\alpha^{-1} \beta d r+\alpha\left(d \bar{t}-a \sin ^{2} \theta d \bar{\phi}\right)\right)^{2} \\
+\rho^{2} d \theta^{2}+\left(r^{2}+a^{2}\right) \sin ^{2} \theta d \bar{\phi}^{2},
\end{gathered}
$$

with

$$
\alpha^{2}=\frac{2 M r}{\rho^{2}}, \quad \beta^{2}=\frac{2 M r}{r^{2}+a^{2}}, \quad \rho^{2}=r^{2}+a^{2} \cos ^{2} \theta,
$$

where $\alpha$ and $\beta$ are the positive roots. The constants $M$ and $a$ are the mass and spin parameter (angular momentum divided by mass) of the black hole, and we use units with $G=c=1$.

The "time" coordinates $t$ and $\bar{t}$ differ from each other only by the addition of a function of $r$, as do the azimuthal angle coordinates $\phi$ and $\bar{\phi}$ :

$$
d \bar{t}=d t+\frac{\beta}{1-\beta^{2}} d r, \quad d \bar{\phi}=d \phi+\frac{\beta}{1-\beta^{2}} \frac{a}{r^{2}+a^{2}} d r
$$

Note that, since the coordinates differ only by functions of $r$, the metric coefficients of all terms not involving $d r$ are equal for the two coordinate systems. In the non-rotating case $a=0, \mathrm{BL}$ becomes Schwarzschild and Doran becomes Painlevé-Gullstrand.

In $\mathrm{BL}$ coordinates the metric has just one off-diagonal $(d t d \phi)$ term, and the constant $t$ surfaces are orthogonal to stationary, zero angular momentum observers. In Doran coordinates, the curves $d \theta=d \bar{\phi}=\beta d r+\alpha^{2} d \bar{t}=0$ 
are infalling timelike geodesics with zero angular momentum, unit energy (at rest at infinity), and proper time $d \bar{t}$. The surfaces of constant $\bar{t}$ are orthogonal to these geodesics. The event horizon of the black hole is located where $\beta=1$, i.e. at

$$
r_{h}=M+\sqrt{M^{2}-a^{2}} .
$$

Doran coordinates are regular at the horizon, but the BL $t$ and $\phi$ coordinates both run to infinity there at constant $\bar{t}$ and $\bar{\phi}$, as can be seen from the coordinate transformation (4). In the extremal case, $a=M$, the horizon is located at $r_{h}=M$. From here on we adopt coordinates with $M=1$.

We are interested in the geometries of the equatorial constant time slices, which are given by

$$
\begin{aligned}
d l_{\mathrm{BL}}^{2} & =\frac{r^{2}}{(r-1-\epsilon)(r-1+\epsilon)} d r^{2}+\left(r^{2}+a^{2}+\frac{2 M a^{2}}{r}\right) d \phi^{2} \\
d l_{\text {Doran }}^{2} & =\frac{r^{2}}{r^{2}+a^{2}} d r^{2}-\sqrt{\frac{8 r}{r^{2}+a^{2}}} d r d \bar{\phi}+\left(r^{2}+a^{2}+\frac{2 M a^{2}}{r}\right) d \bar{\phi}^{2},(7)
\end{aligned}
$$

where $\epsilon=r_{h}-1=\sqrt{1-a^{2}}$ is the deviation of the horizon radius from $r=1$. In the extremal limit we have $\epsilon \rightarrow 0$, and on a BL time slice the radial distance to the bifurcation surface diverges as $\ln \epsilon 2^{2}$

\section{Location of orbits in the extremal limit}

Let us now see how the photon orbit and ISCO can behave as described above. First, it is clear that on the Doran slice, since the limiting metric is regular in the $r$ coordinate at the horizon, the fact that the radial coordinate of these orbits tends to $r_{h}$ means that they must in fact coincide with the horizon. This can also be characterized by the proper time interval along an infalling, zero angular momentum geodesic as these radii are crossed. On such a geodesic the proper time $d \bar{t}$ is related to $d r$ by $d \bar{t}=\left(\beta / \alpha^{2}\right) d r$ [cf. (2)], which at the horizon in the extremal limit becomes $d \bar{t}=d r / 2$. The infalling proper time from the photon orbit or ISCO to the horizon thus goes to zero. This illustrates and verifies the fact that these orbits both coalesce to the horizon generators.

\footnotetext{
${ }^{2}$ This refers to the distance orthogonal to the circles of constant $r$, at constant $\phi$. Note that the two angles $\phi$ and $\bar{\phi}$ wrap infinitely relative to each other as the horizon is approached (4). The distances to the horizon on a BL slice at constant $\bar{\phi}$ and on a Doran slice at constant $\phi$ both diverge logarithmically in the non-extremal case and linearly in the extremal case.
} 
The photon orbit is lightlike, so it is not surprising that it can converge to the horizon generators, but the ISCO is timelike. Evidently the 4-velocity of the ISCO must diverge as a vector in this limit. In fact, using the results of Ref. [1] with help from Mathematica, one finds that the Doran components of the ISCO 4-velocity are given by

$$
\begin{aligned}
& \frac{d \bar{t}}{d s}=\frac{2^{5 / 3}}{\sqrt{3}} \epsilon^{-2 / 3}-\frac{\sqrt{3}}{2}+O\left(\epsilon^{2 / 3}\right) \\
& \frac{d \bar{\phi}}{d s}=\frac{2^{2 / 3}}{\sqrt{3}} \epsilon^{-2 / 3}-\frac{3 \sqrt{3}}{4}+O\left(\epsilon^{2 / 3}\right) .
\end{aligned}
$$

(The BL components are the same, since the orbit lies at constant $r$.) Despite the divergence in the 4 -velocity, the energy and angular momentum per unit mass tend to the finite values $1 / \sqrt{3}$ and $2 / \sqrt{3}$ respectively $\left.\right|^{3}$ The limiting frequency shift factor of a photon emitted from the ISCO in the transverse direction, i.e. orthogonal to the orbit, is $(d t / d s)^{-1} \sim \epsilon^{2 / 3}$. By this redshift a distant observer could in principle infer that the orbit coincides with the horizon in the extremal limit 4 As mentioned in the introduction, the limiting velocity of the ISCO relative to the "locally non-rotating frame" is $1 / 2$ [1]. The 4 -velocity of that frame also stretches to an infinite vector and approaches the horizon generator, so the fact that this relative velocity is less than 1 is not inconsistent with the ISCO approaching the speed of light.

Now let us examine the distance between the orbits on a BL slice. The perpendicular distance between circles of two radii $r_{1,2}=1+\delta_{1,2}$ on the $\mathrm{BL}$ slice (6) is given by

$$
\Delta l=\left(\sqrt{x^{2}-\epsilon^{2}}+\ln \left[2\left(x+\sqrt{x^{2}-\epsilon^{2}}\right]\right)_{\delta_{1}}^{\delta_{2}},\right.
$$

where $x=r-1$. If $\delta_{2}$ remains finite and $\delta_{1}, \epsilon \rightarrow 0$, then the distance $\Delta l$ diverges as $\ln \delta_{1}$. If both $\delta_{1}, \delta_{2} \rightarrow 0$, then the distance depends on precisely how they approach zero. Suppose that $\delta_{1}=c_{1} \epsilon^{p_{1}}$ and $\delta_{2}=c_{2} \epsilon^{p_{2}}$, where $c_{1,2}>0$ and $0<p_{2} \leq p_{1} \leq 1$ are constants. Then we have

$$
\lim _{\epsilon \rightarrow 0} \Delta l= \begin{cases}\left(p_{2}-p_{1}\right) \ln \epsilon & p_{2}<p_{1} \\ \ln \left(c_{2} / c_{1}\right) & p_{2}=p_{1}<1 \\ \ln \left[\frac{c_{2}+\sqrt{c_{2}^{2}-1}}{c_{1}+\sqrt{c_{1}^{2}-1}}\right] & p_{2}=p_{1}=1\end{cases}
$$

\footnotetext{
${ }^{3}$ To obtain the $O\left(\epsilon^{0}\right)$ terms in $(89)$ it is necessary to expand the ISCO radius out to $O\left(\epsilon^{8 / 3}\right)$. The finite terms are needed if the energy and angular momentum are computed directly using the 4-velocity.

${ }^{4}$ The convergence is slow however: a spin parameter $a=0.998$ (which corresponds to $\epsilon \sim 0.06$ ) yields horizon radius $\sim 1.06$, photon orbit radius $\sim 1.07$, ISCO radius $\sim 1.24$, and ISCO redshift factor $\sim 0.09$.
} 
In particular, according to Ref [1] the radial coordinate for the photon orbit is $r_{\mathrm{ph}} \approx 1+(2 / \sqrt{3}) \epsilon$, and for the ISCO it is $r_{\text {isco }} \approx 1+2^{1 / 3} \epsilon^{2 / 3}$. Thus the limiting distance from the photon orbit to the bifurcation surface is $\Delta l\left(r_{h}, r_{\mathrm{ph}}\right)=(1 / 2) \ln 3$, the distance from the ISCO to the bifurcation surface diverges as $\Delta l\left(r_{h}, r_{\text {isco }}\right) \sim-(1 / 3) \ln \epsilon$, and the distance from the ISCO to the ergosurface at $r=2$ diverges as $\Delta l\left(r_{\text {isco }}, r_{\text {ergo }}\right) \sim-(2 / 3) \ln \epsilon$.

In conclusion, the orbits both coincide and do not coincide, depending on where they are examined. This is possible since in the extremal limit there is an infinite stretching of the spacetime region outside the horizon along a constant BL time slic $5^{5}$, and no such stretching on a horizon-crossing time slice.

\section{Acknowledgements}

I thank E. Barausse, A. Buonanno, and E. Poisson for useful comments on a draft of this paper. This work was supported in part by NSF grant PHY-0903572.

\section{References}

[1] J. M. Bardeen, W. H. Press, S. A. Teukolsky, "Rotating black holes: Locally nonrotating frames, energy extraction, and scalar synchrotron radiation," Astrophys. J. 178, 347 (1972).

[2] C. Doran, "A New form of the Kerr solution," Phys. Rev. D61, 067503 (2000) gr-qc/9910099.

[3] J. M. Bardeen and G. T. Horowitz, "The Extreme Kerr throat geometry: A Vacuum analog of $\mathrm{AdS}_{2} \times \mathrm{S}^{2}$," Phys. Rev. D 60, 104030 (1999) arXiv:hep-th/9905099.

\footnotetext{
${ }^{5}$ The extremal metric in the neighborhood of the bifurcation surface is written in Ref. 3 using rescaled time and radius coordinates $T=\lambda t, R=(r-M) / \lambda$, and shifted angle $\Phi=\phi-t / 2 M$, and taking the limit $\lambda \rightarrow 0$. For that metric one can show that there are marginally stable orbits at all radii, all with the same energy and angular momentum as that of the extremal ISCO.
} 